\title{
Quantum statistical properties of the radiation field in a cavity with a movable mirror
}

\author{
C. Brif ${ }^{1,2}$ and A. Mann ${ }^{2}$ \\ ${ }^{1}$ LIGO Project, California Institute of Technology, Pasadena, CA 91125 \\ ${ }^{2}$ Department of Physics, Technion-Israel Institute of Technology, Haifa 32000, Israel
}

\begin{abstract}
A quantum system composed of a cavity radiation field interacting with a movable mirror is considered and quantum statistical properties of the field are studied. Such a system can serve in principle as an idealized meter for detection of a weak classical force coupled to the mirror which is modelled by a quantum harmonic oscillator. It is shown that the standard quantum limit on the measurement of the mirror position arises naturally from the properties of the system during its dynamical evolution. However, the force detection sensitivity of the system falls short of the corresponding standard quantum limit. We also study the effect of the nonlinear interaction between the moving mirror and the radiation pressure on the quadrature fluctuations of the initially coherent cavity field.
\end{abstract}

42.50.Dv, 03.65.Bz, 04.80.Nn

\section{INTRODUCTION}

Due to recent experimental efforts to detect gravitational waves [1], the problem of fundamental limitations on the detection sensitivity imposed by the quantum nature of the measurement device has become of practical importance. The research during the last decade focused mostly on interferometric schemes in which the relative shift in the positions of two end mirrors, caused by a gravitational wave, results in a phase shift between the light beams in the two arms. This phase shift can be detected by measuring the output light. The first analysis of quantum limitations on the measurement sensitivity in such an interferometric scheme was made by Caves [2] in the early eighties. According to this analysis, there are two sources of quantum noise: the photon-counting noise and the radiation-pressure noise. When these two sources of noise are balanced by adjusting the light intensity, the sensitivity achieves the so-called standard quantum limit (SQL).

More recently, Pace et al. 3] studied quantum limitations on the interferometer sensitivity using a more detailed analysis based on a Hamiltonian description of the interaction of light with the movable end mirrors. Some other works [4] were devoted to the study of quantum noise reduction in simpler optical systems: a light beam reflected by a movable mirror or a single Fabry-Pérot cavity with one movable mirror. Assuming high light intensities, the above works mostly employed semi-classical calculational techniques, with the quantum fluctuations of the light field considered as a small addition to the large classical amplitude. The quantum theory of the radiationpressure fluctuations on a mirror was also studied in [8].

In the present paper we consider a simple but nevertheless instructive system, consisting of a cavity radiation field interacting with a movable mirror 9 [1]. As was shown recently [12,13], such a system can be utilized to generate a variety of non-classical states (especially, the so-called Schrödinger-cat states) of both the cavity field and the mirror. We employ the fully quantum description of the system and study quantum statistical properties of the cavity field. While the photon statistics of the field does not change during the interaction, the phase distribution and quadrature fluctuations exhibit interesting dynamics.

If the mirror (modelled by a quantum harmonic oscillator) is driven by an external classical force, this will result in a phase shift of the cavity mode. We study quantum limitations imposed on the detection of the force by the intrinsic quantum uncertainty in a measurement of this phase shift. It is satisfactory to find that the SQL for the measurement of the mirror position naturally emerges from the dynamical properties of the system. On the other hand, the sensitivity of the force detection in this scheme does not reach the corresponding SQL. We also show that analytical approximations made to describe phase properties of the field should work very well for light intensities needed to achieve the SQL in appropriate experimental setups.

\section{THE STANDARD QUANTUM LIMIT IN THE SIMPLEST OPTOMECHANICAL SENSOR}

As is well known, the SQL arises due to the Heisenberg uncertainty relation for the position and momentum of the movable mirror. This limit can be understood conceptually by considering a simple measurement scheme consisting of a movable mirror (modelled, for example, by a linear harmonic oscillator of mass $m$ and angular frequency $\left.\omega_{m}\right)$ and a monochromatic light beam of angular frequency $\omega_{0}$. A shift $z$ in the mirror position, caused by an external classical force (e.g., by a gravitational wave), results in a phase shift $\phi=2 \omega_{0} z / c$ of the reflected light beam (here $c$ is the velocity of light). Such a scheme is referred to as an optomechanical sensor. The experimental progress towards the observation of quantum limits in such a sensor was reported recently in [14]. For a recent theoretical treatment of such a scheme, including a detailed analysis of various sources of noise, see [15]. 
If the incident light beam is in a coherent state with mean photon number $\bar{N}$, the phase uncertainty is $\Delta \phi \simeq$ $1 /\left(2 \bar{N}^{1 / 2}\right)$ (provided that $\left.\bar{N} \gg 1\right)$. This phase-fluctuation noise results in the uncertainty $(\Delta z)_{\mathrm{pf}} \simeq c /\left(4 \omega_{0} \bar{N}^{1 / 2}\right)$ in the measurement of the mirror position. Increasing the light intensity, one can suppress the phase-fluctuation noise, but this leads to increased fluctuations in the radiation pressure which on the average transfers the momentum $\left(2 \hbar \omega_{0} / c\right) \bar{N}$ to the mirror. These radiationpressure fluctuations result in the mirror position uncertainty $(\Delta z)_{\mathrm{rp}} \simeq 2 \hbar \omega_{0} \bar{N}^{1 / 2} /\left(m c \omega_{m}\right)$. Optimizing the sum $(\Delta z)^{2}=(\Delta z)_{\mathrm{pf}}^{2}+(\Delta z)_{\mathrm{rp}}^{2}$ as a function of $\bar{N}$, one finds the uncertainty $(\Delta z)_{\text {opt }}$ which is $\sqrt{2}$ times the SQL for a harmonic oscillator,

$$
(\Delta z)_{\mathrm{SQL}}=\sqrt{\hbar /\left(2 m \omega_{m}\right)} .
$$

The optimum mean number of photons is

$$
\bar{N}_{\mathrm{opt}}=m \omega_{m} c^{2} /\left(8 \hbar \omega_{0}^{2}\right)
$$

In many applications (e.g., for detection of gravitational waves) it makes sense to consider what limitations apply due to the quantum nature of the measuring apparatus on the detection of a weak classical force coupled to the oscillator 16. Consider a constant force $F$ that acts on the mirror during time $t$ which is much shorter than the oscillator period $2 \pi / \omega_{m}$. In order to detect this force, the momentum $p_{F} \simeq F t$ it transfers to the mirror should be larger than the SQL for the measurement of the oscillator momentum, $(\Delta p)_{\mathrm{SQL}}=\left(\hbar m \omega_{m} / 2\right)^{1 / 2}$. Correspondingly, the minimum detectable force is

$$
F_{\mathrm{SQL}}=t^{-1}(\Delta p)_{\mathrm{SQL}}=t^{-1} \sqrt{\hbar m \omega_{m} / 2}
$$

It was argued $\sqrt{17} 20$ that the SQL on the detection of a weak classical force (which causes the displacement of the movable mirror in the sensor) can be surpassed using correlations between the phase-fluctuation noise and the radiation-pressure noise. In particular, it was predicted [4] that amplitude-phase correlations, created by the nonlinear interaction between the movable mirror and the radiation pressure, will produce squeezing in the reflected beam. Consequently, it was proposed [4] to use homodyne detection of the squeezed quadrature in order to compensate for the radiation-pressure noise, thereby making it possible to overcome the SQL with high enough light intensity.

\section{THE MODEL}

The solution of the wave equation for the radiation field in a cavity with moving boundaries is in general a very complicated problem even in the classical case [9, 10]. However, in the adiabatic approximation, when the amplitude and frequency of the mirror motion are much smaller than the wavelength and frequency of the cavity mode, the dynamics of the system can be described by an effective Hamiltonian [1]. Of course, all phenomena associated with the dynamical Casimir effect (e.g., the resonant generation of photons) are neglected in this description.

The free Hamiltonian for the system of the radiation field in a linear cavity with one movable mirror is

$$
H_{0}=H_{c}+H_{m}=\hbar \omega_{c} a^{\dagger} a+\hbar \omega_{m} b^{\dagger} b
$$

where $\omega_{c}$ and $a$ are the angular frequency and the boson annihilation operator of the cavity mode, while $\omega_{m}$ and $b$ are the angular frequency and the annihilation operator of the movable mirror modelled by a quantum harmonic oscillator. For a cavity of free length $L$, one has $\omega_{c}=\pi(c / L) n, n \in \mathbb{N}$. If the mirror displacement from the equilibrium position is $z$, then the cavity length changes $L \rightarrow L+z$, and the cavity angular frequency changes as

$$
\omega_{c} \rightarrow \pi \frac{c}{L+z} n \approx \omega_{c}\left(1-\frac{z}{L}\right)
$$

Therefore, the effective interaction Hamiltonian reads

$$
H_{\mathrm{int}}=-\hbar \omega_{c} \frac{z}{L} a^{\dagger} a=-\hbar g a^{\dagger} a\left(b+b^{\dagger}\right),
$$

where

$$
\begin{aligned}
& z=\left(\frac{\hbar}{2 m \omega_{m}}\right)^{1 / 2}\left(b+b^{\dagger}\right), \\
& g=\frac{\omega_{c}}{L}\left(\frac{\hbar}{2 m \omega_{m}}\right)^{1 / 2} .
\end{aligned}
$$

Here, $m$ is the mass of the movable mirror. If, in addition, we assume that the mirror is driven by a classical external time-dependent force $F(t)$, the corresponding part of the Hamiltonian is given by

$$
\begin{aligned}
& H_{F}=-F(t) z=-\hbar f(t)\left(b+b^{\dagger}\right), \\
& f(t)=\left(2 m \omega_{m} \hbar\right)^{-1 / 2} F(t) .
\end{aligned}
$$

Then the total Hamiltonian $H_{\text {tot }}=H_{0}+H_{\text {int }}+H_{F}$ reads

$H_{\mathrm{tot}}=\hbar \omega_{c} a^{\dagger} a+\hbar \omega_{m} b^{\dagger} b-\hbar g a^{\dagger} a\left(b+b^{\dagger}\right)-\hbar f(t)\left(b+b^{\dagger}\right)$.

It is known that the time evolution operator for this system can be written in a closed form 12,13. One can easily verify that this evolution operator is given by

$U(\tau)=\exp \left(-i r a^{\dagger} a \tau\right) \exp \left[-i b^{\dagger} b \tau+i\left(k a^{\dagger} a+\lambda\right)\left(b+b^{\dagger}\right) \tau\right]$.

With some algebra $[13$, this operator can be rewritten as

$$
\begin{aligned}
U(\tau)= & \exp \left(-i r a^{\dagger} a \tau\right) \exp \left[i \mu\left(k a^{\dagger} a+\lambda\right)^{2}\right] \\
& \times \exp \left[\left(k a^{\dagger} a+\lambda\right)\left(\eta b^{\dagger}-\eta^{*} b\right)\right] \exp \left(-i b^{\dagger} b \tau\right) .
\end{aligned}
$$


Here, $\tau=\omega_{m} t$ is the scaled time and other scaled parameters are $r=\omega_{c} / \omega_{m}$ and $k=g / \omega_{m}$. Also, $\lambda, \mu$, and $\eta$ are dimensionless functions of time:

$$
\begin{aligned}
\lambda(\tau) & =\frac{1}{\tau} \int_{0}^{\tau / \omega_{m}} f\left(t^{\prime}\right) d t^{\prime}, \\
\mu(\tau) & =\tau-\sin \tau, \\
\eta(\tau) & =1-\exp (-i \tau) .
\end{aligned}
$$

An interesting feature of this system is that for times $\tau=2 \pi, 4 \pi, \ldots$ (i.e., after a full cycle of the mirror oscillatory motion), one finds $\eta=0$, and the field and mirror subsystems become fully disentangled (if they were uncorrelated initially). Furthermore, at these moments, the mirror returns to its initial state. This effect is quite general as it does not depend on the initial states of the subsystems. Another interesting property is that the photon statistics of the cavity field does not change during the interaction.

The existence of the closed form (12) for the evolution operator can be understood if we note that the Hamiltonian (11) is given by a linear combination of operators which close an algebra. First, recall that a solvable Lie algebra (the so-called oscillator algebra) is closed by the four operators $\left\{N_{b}, b, b^{\dagger}, I\right\}$, where $N_{b}=b^{\dagger} b$ is the number operator and $I$ is the identity operator. Non-vanishing commutation relations are

$$
\left[N_{b}, b\right]=-b, \quad\left[N_{b}, b^{\dagger}\right]=b^{\dagger}, \quad\left[b, b^{\dagger}\right]=I .
$$

Alternatively, one can choose for the oscillator algebra the fully Hermitian basis $\left\{N_{b}, X_{b}, Y_{b}, I\right\}$, where $X_{b}=$ $2^{-1 / 2}\left(b+b^{\dagger}\right)$ and $Y_{b}=2^{-1 / 2}\left(b-b^{\dagger}\right) / i$ are the scaled position and momentum operators. Non-vanishing commutation relations are

$$
\left[N_{b}, X_{b}\right]=-i Y_{b}, \quad\left[N_{b}, Y_{b}\right]=i X_{b}, \quad\left[X_{b}, Y_{b}\right]=i I .
$$

It is straightforward to see that the oscillator algebra can also be constructed using two bosonic modes $a$ and $b$. The Hermitian basis is given in the two-mode realization by $\left\{N_{b}, N_{a} X_{b}, N_{a} Y_{b}, N_{a}^{2}\right\}$, where $N_{a}=a^{\dagger} a$ is the number operator for the mode $a$, and non-vanishing commutation relations are

$$
\begin{aligned}
& {\left[N_{b}, N_{a} X_{b}\right]=-i N_{a} Y_{b}, \quad\left[N_{b}, N_{a} Y_{b}\right]=i N_{a} X_{b},} \\
& {\left[N_{a} X_{b}, N_{a} Y_{b}\right]=i N_{a}^{2} .}
\end{aligned}
$$

More generally, it is also possible to consider a solvable Lie algebra which is closed by the eight operators $\left\{N_{b}, N_{a} X_{b}, N_{a} Y_{b}, X_{b}, Y_{b}, N_{a}^{2}, N_{a}, I\right\}$ with non-vanishing commutation relations given by the combination of (18), (19) and

$$
\left[N_{a} X_{b}, Y_{b}\right]=\left[X_{b}, N_{a} Y_{b}\right]=i N_{a}
$$

Since the Hamiltonian (11) is given by a linear combination of the operators $N_{a}, N_{b}, N_{a} X_{b}$, and $X_{b}$, the BakerCampbell-Hausdorff theorem [21] assures the existence of a closed expression for the time evolution operator.

\section{THE EVOLUTION OF COHERENT STATES}

Let us consider a situation in which both the cavity field and the mirror oscillator are initially prepared in coherent states with coherent amplitudes $\alpha$ and $\beta$, respectively:

$$
|\Psi(0)\rangle=|\alpha\rangle_{c} \otimes|\beta\rangle_{m}=e^{-|\alpha|^{2} / 2} \sum_{n=0}^{\infty} \frac{\alpha^{n}}{\sqrt{n !}}|n\rangle_{c} \otimes|\beta\rangle_{m} .
$$

In fact, a quite reasonable choice is to assume that the mirror is initially in the vacuum state (i.e., $\beta=0$ ), but first we will consider an arbitrary $\beta$ for the sake of generality. Acting on the initial state (21) with the evolution operator (13), one obtains:

$$
\begin{aligned}
|\Psi(\tau)\rangle= & e^{-|\alpha|^{2} / 2} \sum_{n=0}^{\infty} \frac{\left(\alpha e^{-i r \tau}\right)^{n}}{\sqrt{n !}} e^{i \mu(k n+\lambda)^{2}}|n\rangle_{c} \\
& \otimes\left[e^{(k n+\lambda)\left(\eta b^{\dagger}-\eta^{*} b\right)}\left|\beta e^{-i \tau}\right\rangle_{m}\right] .
\end{aligned}
$$

Utilizing properties of the displacement operators [22], the state of the system may be written as (we omit an unimportant overall phase factor)

$$
\begin{aligned}
|\Psi(\tau)\rangle= & e^{-|\alpha|^{2} / 2} \sum_{n=0}^{\infty} \frac{\left[\alpha e^{-i \zeta(\tau)}\right]^{n}}{\sqrt{n !}} \\
& \times e^{i \mu(\tau) k^{2} n^{2}}|n\rangle_{c} \otimes\left|\gamma_{n}(\tau)\right\rangle_{m} .
\end{aligned}
$$

In the reference frame rotating with the field frequency $\omega_{c}$, the phase $\zeta(\tau)$ is given by

$$
\zeta(\tau)=2 k \lambda(\tau) \mu(\tau)+k \operatorname{Im}[\beta \eta(\tau)] .
$$

In Eq. (23), $\left|\gamma_{n}(\tau)\right\rangle$ is a coherent state with the amplitude

$$
\gamma_{n}(\tau)=\beta e^{-i \tau}+[k n+\lambda(\tau)] \eta(\tau) .
$$

For $\tau=2 \pi, 4 \pi, \ldots$, the dependence of this amplitude on $n$ disappears, and the system returns to an uncorrelated product state.

In what follows we will be interested in quantum statistical properties of the cavity field. These properties can be calculated from the reduced density matrix, obtained by tracing out the mirror degrees of freedom,

$$
\begin{aligned}
\rho_{c}(\tau)= & \operatorname{Tr}_{m}\{|\Psi(\tau)\rangle\langle\Psi(\tau)|\} \\
= & e^{-|\alpha|^{2}} \sum_{n=0}^{\infty} \sum_{n^{\prime}=0}^{\infty} \frac{\left(\alpha e^{i \zeta}\right)^{n}\left(\alpha^{*} e^{-i \zeta}\right)^{n^{\prime}}}{\sqrt{n ! n^{\prime} !}} \\
& \times e^{i \mu k^{2}\left(n^{2}-n^{\prime 2}\right)}\left\langle\gamma_{n^{\prime}} \mid \gamma_{n}\right\rangle|n\rangle_{c c}\left\langle n^{\prime}\right|,
\end{aligned}
$$

where

$$
\begin{aligned}
\left\langle\gamma_{n^{\prime}} \mid \gamma_{n}\right\rangle & =\exp \left(\gamma_{n^{\prime}}^{*} \gamma_{n}-\frac{1}{2}\left|\gamma_{n}\right|^{2}-\frac{1}{2}\left|\gamma_{n^{\prime}}\right|^{2}\right) \\
& =\exp \left[-\dot{\mu} k^{2}\left(n-n^{\prime}\right)^{2}\right] \quad \text { for } \beta=0 .
\end{aligned}
$$

Note that $\dot{\mu}=\frac{1}{2}|\eta|^{2}=1-\cos \tau$. Obviously, the Poissonian photon statistics of the cavity field is preserved during the evolution. 


\section{PHASE PROPERTIES OF THE CAVITY FIELD}

As can be seen from equations (23) and (24), the external force acting on the mirror produces a phase shift of the cavity mode. At least in principle, this phase shift can be measured, thereby revealing information about the classical force. Accuracy of such a measurement will inevitably depend on the phase properties of the radiation mode, because an externally induced phase shift is detectable only if it is larger than the intrinsic phase uncertainty of the field.

\section{A. Canonical and realistic phase distributions}

In quantum mechanics, one can describe a measurement by the corresponding positive operator-valued measure (POVM) 23]. The so-called canonical POVM for an idealized phase measurement is given by [24,25.

$$
\Pi_{\mathrm{can}}(\theta)=\frac{1}{2 \pi}|\theta\rangle\langle\theta|, \quad| \theta\rangle=\sum_{n=0}^{\infty} e^{i n \theta}|n\rangle .
$$

The corresponding phase distribution for the cavity field is

$$
P_{\text {can }}(\theta)=\operatorname{Tr}\left[\Pi_{\text {can }}(\theta) \rho_{c}\right]=(2 \pi)^{-1}\left\langle\theta\left|\rho_{c}\right| \theta\right\rangle .
$$

Substituting expression (26) for $\rho_{c}$ into (30), we obtain

$$
\begin{aligned}
P_{\text {can }}(\theta)= & \frac{e^{-|\alpha|^{2}}}{2 \pi} \sum_{n=0}^{\infty} \sum_{n^{\prime}=0}^{\infty} \frac{|\alpha|^{n+n^{\prime}}}{\sqrt{n ! n^{\prime} !}} e^{-i\left(\theta-\zeta-\varphi_{\alpha}\right)\left(n-n^{\prime}\right)} \\
& \times e^{i \mu k^{2}\left(n^{2}-n^{\prime 2}\right)}\left\langle\gamma_{n^{\prime}} \mid \gamma_{n}\right\rangle,
\end{aligned}
$$

where $\varphi_{\alpha}=\arg (\alpha)$. For $\beta=0$, we can use expression (28) for $\left\langle\gamma_{n^{\prime}} \mid \gamma_{n}\right\rangle$. Then the double summation in Eq. (31) can be rearranged in such a way that $P_{\text {can }}(\theta)$ takes the form of a Fourier series:

$$
P_{\text {can }}(\theta)=\frac{1}{2 \pi} \sum_{q=-\infty}^{\infty} e^{-\dot{\mu} k^{2} q^{2}} \mathcal{A}_{q} e^{i\left(\theta-\zeta-\varphi_{\alpha}\right) q}
$$

where

$$
\begin{aligned}
& \mathcal{A}_{q}=e^{-|\alpha|^{2}} \xi_{q}^{|q|} \sum_{n=0}^{\infty} \frac{\xi_{q}^{2 n}}{\sqrt{n !(n+|q|) !}} \\
& \xi_{q}=|\alpha| \exp \left(-i \mu k^{2} q\right) .
\end{aligned}
$$

The problem of the quantum description of optical phase and the problem of its measurement were widely discussed during the last decade (see, e.g., reviews [26,27). However, existence of an experimental procedure for phase measurement with the canonical POVM (29) is still an open question. On the other hand, a possible way to measure the phase of the radiation field mode is by means of heterodyne detection (see, e.g., [28]). Relating to our model, a possible experimental procedure might be as follows. At some moment $t$ one stops the interaction between the field and the movable mirror (by fixing the mirror position) and lets the radiation leak from the cavity. The output field is detected using the heterodyne scheme, which employs a strong local oscillator highly detuned from the signal. The two Fourier components of the photocurrent are proportional to the quadratures of the cavity field at the moment $t$, which can be used to determine the phase. The POVM for this phase measurement is

$$
\Pi_{Q}(\theta)=\frac{1}{\pi} \int_{0}^{\infty}|A\rangle\langle A| r d r, \quad A=r e^{i \theta},
$$

where $|A\rangle$ is a coherent state with amplitude $A$. The corresponding phase distribution is obtained by integrating the $Q$ distribution function,

$$
Q(A)=\frac{1}{\pi}\left\langle A\left|\rho_{c}\right| A\right\rangle
$$

over the radial coordinate in the complex plane:

$$
P_{Q}(\theta)=\operatorname{Tr}\left[\Pi_{Q}(\theta) \rho_{c}\right]=\int_{0}^{\infty} Q\left(r e^{i \theta}\right) r d r
$$

For the cavity field mode with $\rho_{c}$ of equation (26), we obtain

$$
\begin{aligned}
P_{Q}(\theta)= & \frac{e^{-|\alpha|^{2}}}{2 \pi} \sum_{n=0}^{\infty} \sum_{n^{\prime}=0}^{\infty} \Gamma\left(\frac{n+n^{\prime}+2}{2}\right) \frac{|\alpha|^{n+n^{\prime}}}{n ! n^{\prime} !} \\
& \times e^{-i\left(\theta-\zeta-\varphi_{\alpha}\right)\left(n-n^{\prime}\right)} e^{i \mu k^{2}\left(n^{2}-n^{\prime 2}\right)}\left\langle\gamma_{n^{\prime}} \mid \gamma_{n}\right\rangle .
\end{aligned}
$$

We see that the distributions $P_{\text {can }}(\theta)$ and $P_{Q}(\theta)$ differ by the factor

$$
\frac{1}{\sqrt{n ! n^{\prime} !}} \Gamma\left(\frac{n+n^{\prime}+2}{2}\right)
$$

in the double sum over $n$ and $n^{\prime}$. Correspondingly, $P_{Q}(\theta)$ is usually slightly wider than $P_{\text {can }}(\theta)$. (It has been argued 29] that this widening is related to contamination by external noise during the heterodyne detection.) For $\beta=0$, the rearrangement of the double summation in (38) gives

$$
P_{Q}(\theta)=\frac{1}{2 \pi} \sum_{q=-\infty}^{\infty} e^{-\dot{\mu} k^{2} q^{2}} \mathcal{B}_{q} e^{i\left(\theta-\zeta-\varphi_{\alpha}\right) q}
$$

where

$$
\mathcal{B}_{q}=e^{-|\alpha|^{2}} \xi_{q}^{|q|} \sum_{n=0}^{\infty} \frac{\Gamma\left(n+\frac{1}{2}|q|+1\right)}{\Gamma(n+|q|+1)} \frac{\xi_{q}^{2 n}}{n !},
$$

and $\xi_{q}$ is given by Eq. (34). Note that $\mathcal{B}_{0}=1$. The summation in (41) is proportional to the Kummer series [30], so we can write

$$
\mathcal{B}_{q}=e^{-|\alpha|^{2}} \xi_{q}^{|q|} \frac{\Gamma\left(\frac{1}{2}|q|+1\right)}{\Gamma(|q|+1)} \Phi\left(\frac{1}{2}|q|+1,|q|+1 ; \xi_{q}^{2}\right) .
$$


Here, $\Phi(a, b ; x)$ is the Humbert symbol for the confluent hypergeometric function (the Kummer function). Using properties of special functions, $\mathcal{B}_{q}$ can also be written as

$$
\begin{aligned}
\mathcal{B}_{q}= & \frac{\sqrt{\pi}}{2} \exp \left(-|\alpha|^{2}\right) \exp \left(\xi_{q}^{2} / 2\right) \xi_{q} \\
& \times\left[I_{(|q|-1) / 2}\left(\xi_{q}^{2} / 2\right)+I_{(|q|+1) / 2}\left(\xi_{q}^{2} / 2\right)\right],
\end{aligned}
$$

where $I_{\nu}(x)$ is the modified Bessel function of the first kind. In the strong-field limit $|\alpha|^{2} \gg 1$, we can use the asymptotic expansion for the confluent hypergeometric function or for the modified Bessel function to obtain (for fixed $q$ ):

$$
\mathcal{B}_{q} \approx \exp \left(-|\alpha|^{2}+\xi_{q}^{2}\right)\left(1-\frac{q^{2}}{4 \xi_{q}^{2}}+\cdots\right) .
$$

For high field intensities, the behavior of both phase distributions is qualitatively very similar. In what follows we will consider the $P_{Q}(\theta)$ distribution, because of two reasons: (i) phase measurements with the corresponding POVM $\Pi_{Q}(\theta)$ are experimentally feasible and (ii) the existence of a closed expression for $\mathcal{B}_{q}$ gives a calculational advantage.

Let us assume the phase range $-\pi \leq \theta<\pi$. Then moments of the phase distribution are determined by

$$
\bar{\theta}=\int_{-\pi}^{\pi} \theta P_{Q}(\theta) d \theta, \quad \overline{\theta^{2}}=\int_{-\pi}^{\pi} \theta^{2} P_{Q}(\theta) d \theta .
$$

A simple calculation gives

$$
\begin{gathered}
\bar{\theta}=2 \sum_{q=1}^{\infty} \frac{1}{q} e^{-\dot{\mu} k^{2} q^{2}} \operatorname{Im}\left[\mathcal{B}_{q} e^{-i\left(\zeta+\varphi_{\alpha}+\pi\right) q}\right], \\
\overline{\theta^{2}}=\frac{\pi^{2}}{3}+4 \sum_{q=1}^{\infty} \frac{1}{q^{2}} e^{-\dot{\mu} k^{2} q^{2}} \operatorname{Re}\left[\mathcal{B}_{q} e^{-i\left(\zeta+\varphi_{\alpha}+\pi\right) q}\right] .
\end{gathered}
$$

Using these expressions, we can calculate the phase uncertainty, $\Delta \theta=\left(\overline{\theta^{2}}-\bar{\theta}^{2}\right)^{1 / 2}$. This can be done numerically, but some important information may also be deduced from an analytical approximation.

\section{B. Analytical approximations and numerical results}

In our considerations we neglected losses which occur in a realistic case due to the relaxation of the cavity field and the dissipation of the mirror oscillator. For a cavity with a very high finesse and a mirror oscillator with a very high quality factor, neglecting the losses can be a fair approximation for short interaction times, say, for $t<10^{-3}$ s. Therefore, for low-frequency oscillators (with $\omega_{m} \sim 2 \pi$ $\mathrm{Hz}$, as in the LIGO interferometric gravitational-wave detectors (1), it is reasonable to consider $\tau<10^{-2}$. For such small values of $\tau$ we can use approximate expressions for $\mu(\tau)$ and $\dot{\mu}(\tau)$. The leading term in $\dot{\mu}(\tau)$ is $\tau^{2} / 2$, so the terms in the series 40 are weighted by the prefactor $\exp \left(-k^{2} \tau^{2} q^{2} / 2\right)$. Correspondingly, the contribution of terms with $q$ much larger than $q_{m}=(k \tau)^{-1}$ is rather insignificant. Then, for high field intensities (i.e., $|\alpha|$ much larger than $q_{m}$ and $|\alpha|^{2} \gg 1$ ), we may approximate $\mathcal{B}_{q}$ by just the leading term in its asymptotic expansion (44). With all these assumptions, we derive

$$
\begin{gathered}
P_{Q}(\theta) \approx \frac{1}{2 \pi} \sum_{q=-\infty}^{\infty} \exp \left[-\frac{1}{2} \sigma^{2} q^{2}\right] \exp [i(\theta-\tilde{\theta}) q], \\
\sigma=k \tau \sqrt{1+\varepsilon_{1}}, \quad \tilde{\theta}=\zeta+\varphi_{\alpha}+\varepsilon_{2}, \\
\varepsilon_{1}=\frac{k^{2} \tau^{4}|\alpha|^{2}}{9}-\frac{\tau^{2}}{12}+\frac{\tau^{4}}{360}, \quad \varepsilon_{2}=\frac{k^{2} \tau^{3}|\alpha|^{2}}{3} .
\end{gathered}
$$

We see that the phase distribution $P_{Q}(\theta)$ is approximated by a Fourier series with Gaussian coefficients,

$$
\exp \left[-\frac{1}{2} \sigma^{2} q^{2}\right]=\int_{-\pi}^{\pi} P_{Q}(\theta) e^{-i(\theta-\tilde{\theta}) q} d \theta .
$$

Though we did not find a closed expression for the series (48), a further approximation may be made. It is clear that the width of the distribution is of the order of $\sigma$ and it is centered near $\tilde{\theta}$. When the distribution is very narrow (i.e., $\sigma \ll 1$ ) and its center is far from the boundaries of the interval $[-\pi, \pi)$ (in comparison with the distribution width $\sigma$ ), it is possible to extend the integration range in (51) from $-\infty$ to $+\infty$ without changing the result significantly. This is equivalent to the replacement of the Fourier series in (48) by the Fourier integral. Formally, we can apply the Poisson summation formula (see, e.g., [31]) to the series (48) to obtain

$$
P_{Q}(\theta) \approx\left(2 \pi \sigma^{2}\right)^{-1 / 2} \sum_{m=-\infty}^{\infty} \exp \left[-\frac{1}{2}(\theta-\tilde{\theta}-2 m \pi)^{2} / \sigma^{2}\right] .
$$

For $\sigma \ll 1$, the series (52) converges much faster than (48). If only the leading term in (52) is left, the phase distribution is approximated by just the Gaussian,

$$
P_{Q}(\theta) \approx\left(2 \pi \sigma^{2}\right)^{-1 / 2} \exp \left[-\frac{1}{2}(\theta-\tilde{\theta})^{2} / \sigma^{2}\right],
$$

centered about $\tilde{\theta}$ and with the dispersion $\sigma$.

The accuracy of our approximations may be verified by calculating numerically the mean phase $\bar{\theta}$ and the phase uncertainty $\Delta \theta$ from the distributions (40) and (48). For simplicity, we took $\zeta=\varphi_{\alpha}=0$ in these calculations. Figure 1 shows how the phase uncertainty $\Delta \theta$ depends on the intensity of the cavity field. The approximation (48) works extremely well for sufficiently large field intensities. The Gaussian approximation $(\sqrt{53})$ is also excellent. In principle, $\Delta \theta$ obtained from (48) coincides with 
$\sigma$ except for the neighborhood of points where the mean phase is $\pm \pi \bmod 2 \pi$. The corresponding deterioration of the phase sensitivity is an artifact of the definition of the phase range from $-\pi$ to $\pi$. The dependence of the mean phase $\bar{\theta}$ on $|\alpha|$ is shown in Fig. 2. It is seen that the value $\tilde{\theta}$ obtained in the Gaussian approximation differs very insignificantly from the exact value of $\bar{\theta}$. One can also verify that the peaks in Fig. 1 appear exactly at the points for which $\bar{\theta}$ in Fig. 2 approaches $\pi$. Figure 3 shows the dependence of the phase uncertainty $\Delta \theta$ on the scaled measurement time $\tau$. Once again, the approximations work very well.

We should note that the value of $k$ we used $(k=7.0)$ has no special physical meaning. In fact, this value is much larger than one would expect in an experiment. We chose such a large $k$ only in order to deal with smaller values of $|\alpha|$ and thereby avoid overflows in numerical calculations. However, this artificial scaling does not distort the physical behavior of the system. Let us consider what will happen for a realistic value of $k$ (e.g., $k \sim 6 \times 10^{-8}$ for the LIGO detectors). Then one obtains a much larger value of $q_{m}=(k \tau)^{-1}$ (e.g., $q_{m} \sim 3 \times 10^{9}$ for the LIGO detectors with the cavity storage time about $\left.10^{-3} \mathrm{~s}\right)$. Therefore, our approximations might be used, but for very large values of $|\alpha|$. In fact, a simple calculation gives $q_{m}^{2}=(4 L / c t)^{2} \bar{N}_{\text {opt }}$, where $t$ is the measurement time and $\bar{N}_{\text {opt }}$ is the mean photon number needed to achieve the SQL in an optomechanical sensor, as given by Eq. (2). With the LIGO parameters, one obtains $\bar{N}_{\text {opt }} \sim 2.5 \times 10^{21}$, so our approximations will work perfectly for the mean photon numbers $\bar{N}=|\alpha|^{2}$ of the order of $\bar{N}_{\text {opt }}$ and even smaller.

\section{QUANTUM LIMITATIONS}

The principal result is that, in the region of small $\tau$, the phase uncertainty $\Delta \theta$ is limited from below by the value $k \tau$ (in fact, this limit is slightly higher due to the factor $\sqrt{1+\varepsilon_{1}}$ in $\sigma$, but this small correction is not important for understanding the basic behavior of the system). Now, let us consider what this phase uncertainty means regarding the measurement of the mirror shift. A phase shift $\phi$ produced due to the mirror position shift $z$ is given by $\phi \simeq\left(2 B \omega_{c} / c\right) z$, where

$$
B=\frac{t c}{2 L}=\frac{\tau c}{2 \omega_{m} L}
$$

is the effective number of bounces by light on the movable mirror during the measurement time $t$. If the uncertainty of the phase shift measurement is $\Delta \theta$, the corresponding uncertainty of the mirror position shift is

$$
\Delta z \simeq \frac{c}{2 B \omega_{c}} \Delta \theta=\frac{\omega_{m} L}{\omega_{c} \tau} \Delta \theta
$$

Since in our model the phase uncertainty $\Delta \theta$ is limited from below by $k \tau$, the corresponding limit on the position shift uncertainty is

$$
(\Delta z)_{\min } \simeq \frac{\omega_{m} L}{\omega_{c} \tau} k \tau=\frac{g L}{\omega_{c}}=\sqrt{\frac{\hbar}{2 m \omega_{m}}},
$$

which is exactly the SQL for the position measurement of a harmonic oscillator.

The limitation on the detection of an external classical force $F(t)$ will depend, of course, not only on the uncertainty $\Delta \theta$ of the phase measurement, but also on the value of the phase shift $\zeta_{F}=2 k \lambda \mu$ produced by this force. The corresponding signal-to-noise ratio is

$$
\frac{S}{N}=\frac{\zeta_{F}(\tau)}{\Delta \theta}=\frac{2 k \lambda(\tau) \mu(\tau)}{\Delta \theta} .
$$

For $\tau \ll 1$, taking for $\Delta \theta$ its lower limit $k \tau$, we obtain

$$
\frac{S}{N} \simeq \frac{k \lambda(\tau) \tau^{3} / 3}{k \tau}=\left(2 \hbar m \omega_{m}\right)^{-1 / 2} \frac{\tau}{3} \int_{0}^{\tau / \omega_{m}} F\left(t^{\prime}\right) d t^{\prime}
$$

Clearly, the sensitivity of the force detection depends on the total phase shift produced by the force during the time $t=\tau / \omega_{m}$. This result just reflects the fact that in our model the information about the system is inferred from a "single-shot" phase measurement at the time $t$, and not from a continuous monitoring of the system. In the case of a force $F$ which is constant during the time $t$, we obtain

$$
\frac{S}{N} \simeq\left(2 \hbar m \omega_{m}\right)^{-1 / 2} \frac{F \tau^{2}}{3 \omega_{m}}
$$

The minimum detectable force $F_{\min }$ is determined from the condition $S / N=1$. Then we obtain

$$
F_{\min } \simeq \sqrt{\frac{18 \hbar m}{\omega_{m} t^{4}}}=\frac{6}{\tau} F_{\mathrm{SQL}}
$$

So, with the method presented here, the minimum detectable force is quite far from the SQL. This result shows that the genuine sensitivity in a specific scheme can differ significantly from the commonly accepted SQL for an abstract oscillator.

\section{QUANTUM FLUCTUATIONS OF THE FIELD QUADRATURES}

It is well known (see, e.g., 32 ) that homodyne detection with a strong local oscillator can be used to measure the rotated field quadrature

$$
X_{\varphi}=a e^{-i \varphi}+a^{\dagger} e^{i \varphi} .
$$

The angle $\varphi$ is the phase of the local oscillator. A quantum state of the signal field $a$ exhibits the phenomenon of squeezing if the uncertainty $\Delta X_{\varphi}=\left(\left\langle X_{\varphi}^{2}\right\rangle-\left\langle X_{\varphi}\right\rangle^{2}\right)^{1 / 2}$ 
is below its vacuum value $\left(\Delta X_{\varphi}\right)_{\mathrm{vac}}=1$ for some angle $\varphi$. Since

$$
X_{\varphi}^{2}=1+2 a^{\dagger} a+a^{2} e^{-2 i \varphi}+a^{\dagger 2} e^{2 i \varphi},
$$

the uncertainty $\Delta X_{\varphi}$ at any time $t$ can be determined by calculating the expectation values of $a$ and $a^{2}$ (recall that the mean number of photons $\left\langle a^{\dagger} a\right\rangle=|\alpha|^{2}$ is constant). For $\beta=0$, a straightforward calculation gives:

$$
\begin{aligned}
\langle a\rangle=\left\langle a^{\dagger}\right\rangle^{*}= & |\alpha| \exp \left[i\left(\zeta+\varphi_{\alpha}+\mu k^{2}\right)\right] \\
& \times \exp \left[-\dot{\mu} k^{2}-|\alpha|^{2}\left(1-e^{2 i \mu k^{2}}\right)\right], \\
\left\langle a^{2}\right\rangle=\left\langle a^{\dagger 2}\right\rangle^{*}= & |\alpha|^{2} \exp \left[2 i\left(\zeta+\varphi_{\alpha}+2 \mu k^{2}\right)\right] \\
& \times \exp \left[-4 \dot{\mu} k^{2}-|\alpha|^{2}\left(1-e^{4 i \mu k^{2}}\right)\right] .
\end{aligned}
$$

Using these results, we can study numerically the behavior of the quadrature uncertainty. Also, in the relevant limits $|\alpha|^{2} \gg 1$ and $\tau \ll 1$, an approximate expression is obtained

$$
\begin{gathered}
\left(\Delta X_{\varphi}\right)^{2} \approx 1+2|\alpha|^{2}\left(1+e^{-2 \Gamma} \cos 2 \vartheta-2 e^{-\Gamma} \cos ^{2} \vartheta\right) \\
\Gamma=k^{2} \tau^{2}\left(1+k^{2}|\alpha|^{2} \tau^{4} / 9\right) \\
\vartheta=\zeta+\varphi_{\alpha}+k^{2}|\alpha|^{2} \tau^{3} / 3-\varphi
\end{gathered}
$$

The variance in Eq. (65) is minimized for $\vartheta=n \pi(n=$ $0, \pm 1, \pm 2, \ldots)$. Then we find

$$
\left(\Delta X_{\varphi}\right)^{2} \approx 1+2|\alpha|^{2}\left(1-e^{-\Gamma}\right)^{2},
$$

which is always larger than 1 .

Figures 4 and 5 show the basic features of the behavior of the quadrature uncertainty. For simplicity, we took $\zeta=\varphi_{\alpha}=0$ in the numerical calculations. In Fig. 4 the dependence of $\Delta X_{\varphi}$ on the homodyne phase $\varphi$ is shown for various field intensities (the range of $\varphi$ from zero to $\pi$ is chosen because $\Delta X_{\varphi}$ is a periodic function of $\varphi$ with the period of $\pi$ ). For the parameters taken, the approximation (65) is excellent, and the quadrature uncertainty $\Delta X_{\varphi}$ reaches a minimum for $\varphi$ about $k^{2}|\alpha|^{2} \tau^{3} / 3$ (i.e., for $\vartheta=0)$. The value of $\Delta X_{\varphi}$ at this minimum is plotted in Fig. 5 versus $|\alpha|$ for several interaction times. Some discrepancy between exact and approximate results appears for small values of $\tau$, but the approximation $(68)$ is excellent for field intensities large enough to assure $\Gamma>1$. For the parameters we considered, no squeezing of the field quadrature was found.

\section{DISCUSSION}

It should be clearly understood that we have no intention to claim that the idealized system discussed in this paper might be immediately used as a practical sensor of weak classical forces (e.g., for detection of gravitational waves). Our study is aimed at understanding the most basic properties of the quantum dynamics of the radiation field in a cavity with a movable mirror. The evolution of the field phase distribution, studied in the framework of a fully quantum approach, is of interest because it helps to clarify how the information about an external force is carried by the light field in much more complicated realistic measurement schemes. In fact, it is very instructive to see how the evolution of the field phase uncertainty determines the sensitivity of the scheme and naturally implies the SQL on the measurement of the mirror position.

It should be emphasized that in our model we assumed that the information about the phase shift accumulated by the cavity field is read by means of a "single-shot" phase measurement. Of course, this only gives information about the integrated effect of an external force during the measurement time. However, if one wants to determine the time dependence of the force, this will require a continuous monitoring of the field phase. In a consistent quantum description, one should take into account that the evolution of the system under the continuous measurement will be seriously affected by the measurementinduced state reduction. A model of continuous broadband measurement for monitoring the position of a free mass was recently analyzed in [33]. It would be also interesting to have such a kind of quantum analysis for the continuous measurement on the system of the radiation field interacting with a movable mirror.

\section{ACKNOWLEDGMENTS}

CB thanks Kip S. Thorne, John Preskill, Yuri Levin, Bill Kells and other participants of the Caltech QND reading group for useful and informative discussions. Financial support from the Lester Deutsch Fund is gratefully acknowledged. This work was supported in part by the Institute of Theoretical Physics at the Department of Physics at the Technion, and CB is grateful to the Institute for hospitality during his visit to the Technion. The LIGO Project is supported by the National Science Foundation under the cooperative agreement PHY-9210038. AM was supported by the Fund for Promotion of Research at the Technion and by the Technion VPR Fund.

[1] Gravitational Waves: Sources and Detectors, edited by I. Ciufolini and F. Fidecaro (World Scientific, Singapore, 1997); Gravitational Wave Detection, edited by K. Tsubono, M. K. Fujimoto, and K. Kuroda (Universal Academy Press, Tokyo, 1997). 
[2] C. M. Caves, Phys. Rev. Lett. 45, 75 (1980); Phys. Rev. D 23, 1693 (1981).

[3] A. F. Pace, M. J. Collett, and D. F. Walls, Phys. Rev. A 47, 3173 (1993).

[4] S. P. Vyatchanin and A. B. Matsko, J. Exp. Theor. Phys. 77, 218 (1993); ibid. 82, 1007 (1996); ibid. 83, 690 (1996).

[5] S. Mancini and P. Tombesi, Phys. Rev. A 49, 4055 (1994).

[6] C. Fabre, M. Pinard, S. Bourzeix, A. Heidmann, E. Giacobino, and S. Reynaud, Phys. Rev. A 49, 1337 (1994).

[7] A. Heidmann and S. Reynaud, Phys. Rev. A 50, 4237 (1994).

[8] P. Samphire, R. Loudon, and M. Babiker, Phys. Rev. A 51, 2726 (1995).

[9] G. T. Moore, J. Math. Phys. 11, 2679 (1970).

[10] M. Janowicz, Phys. Rev. A 57, 4784 (1998).

[11] C. K. Law, Phys. Rev. A 51, 2537 (1995).

[12] S. Mancini, V. I. Man'ko, and P. Tombesi, Phys. Rev. A 55, 3042 (1997).

[13] S. Bose, K. Jacobs, and P. L. Knight, Phys. Rev. A 56, 4175 (1997).

[14] I. Tittonen, G. Breitenbach, T. Kalkbrenner, T. Müller, R. Conradt, S. Schiller, E. Steinsland, N. Blanc, and N. F. de Rooij, Phys. Rev. A 59, 1038 (1999).

[15] K. Jacobs, I. Tittonen, H. M. Wiseman, and S. Schiller, preprint quant-ph/9902040.

[16] C. M. Caves, K. S. Thorne, R. W. P. Drever, V. D. Sandberg, and M. Zimmermann, Rev. Mod. Phys. 52, 341 (1980).

[17] W. G. Unruh, in Quantum Optics, Experimental Gravitation and Measurement Theory, edited by P. Meystre and M. O. Scully (Plenum, New York, 1983), p. 647.
[18] R. S. Bondurant and J. H. Shapiro, Phys. Rev. D 30, 2548 (1984).

[19] M. T. Jaekel and S. Reynaud, Europhys. Lett. 13, 301 (1990).

[20] V. B. Braginsky and F. Ya. Khalili, Quantum Measurement, edited by K. S. Thorne (Cambridge University Press, Cambridge, England, 1992), Chap. VIII.

[21] W. Magnus, Commun. Pure Appl. Math. 7, 649 (1954).

[22] R. J. Glauber, Phys. Rev. 131, 2766 (1963).

[23] C. W. Helstrom, Quantum Detection and Estimation Theory (Academic Press, New York, 1976).

[24] J. H. Shapiro and S. R. Shepard, Phys. Rev. A 43, 3795 (1991).

[25] C. Brif and Y. Ben-Aryeh, Phys. Rev. A 50, 3505 (1994).

[26] R. Tanas, A. Miranowicz, and T. Gantsog, in Progress in Optics, Vol. XXXV, edited by E. Wolf (Elsevier, Amsterdam, 1996), p. 355.

[27] D. T. Pegg and S. M. Barnett, J. Mod. Opt. 44, 225 (1997).

[28] H. M. Wiseman and G. J. Milburn, Phys. Rev. A 47, 1652 (1993).

[29] U. Leonhardt, J. A. Vaccaro, B. Böhmer, and H. Paul, Phys. Rev. A 51, 84 (1995).

[30] A. Erdélyi et al., Bateman Manuscript Project: Higher Transcendental Functions (McGraw-Hill, New York, 1953), Chap. VI.

[31] I. Stakgold, Boundary Value Problems of Mathematical Physics (Macmillan, New York, 1967), Vol. I, pp. 46-47.

[32] H. M. Wiseman, Quantum Semiclass. Opt. 7, 569 (1995).

[33] H. Mabuchi, Phys. Rev. A 58, 123 (1998). 


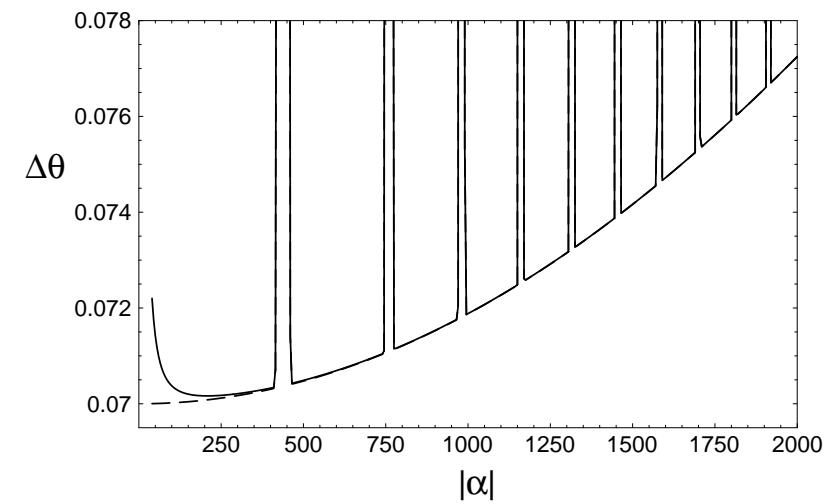

FIG. 1. The phase uncertainty $\Delta \theta$ versus $|\alpha|$ for $k=7.0$ and $\tau=0.01$. The exact result is shown with the solid line and the approximate result, corresponding to the distribution (48), is the dashed line. The difference between exact and approximate results appears only for $|\alpha|<300$. If one ignores peaks, the corresponding curve coincides perfectly with the Gaussian dispersion $\sigma$.

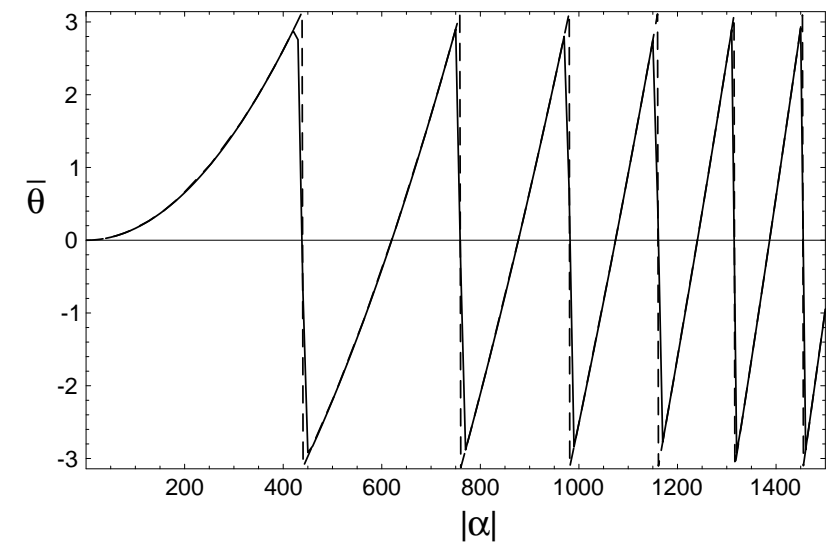

FIG. 2. The mean phase $\bar{\theta}$ versus $|\alpha|$ for $k=7.0$ and $\tau=0.01$. The exact result is shown with a solid line and the Gaussian approximation $\tilde{\theta}=\varepsilon_{2}$ with a dashed line. The difference between exact and approximate results is very insignificant. The mean phase $\bar{\theta}$ obtained from the approximate distribution (48) is not shown here because it is almost indistinguishable from the exact value.

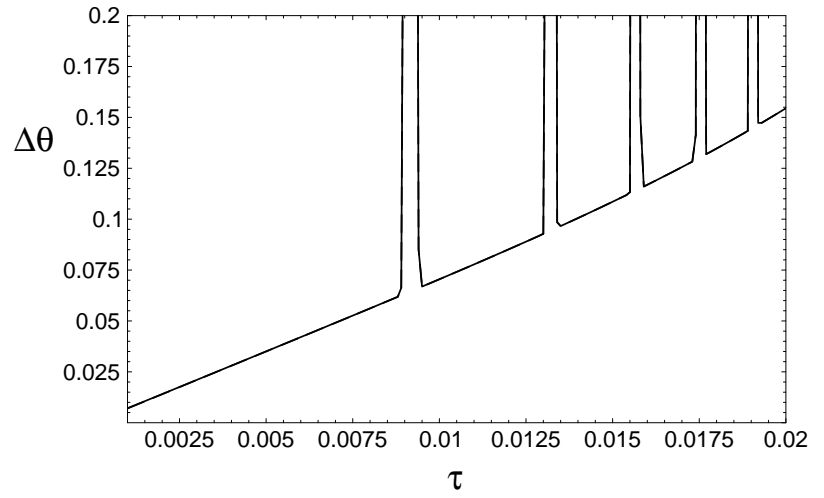

FIG. 3. The phase uncertainty $\Delta \theta$ versus the scaled time $\tau$ for $k=7.0$ and $|\alpha|=500$. The exact uncertainty and the approximate one, corresponding to the distribution (48), are indistinguishable. If one ignores peaks, the corresponding curve coincides perfectly with the Gaussian dispersion $\sigma$.

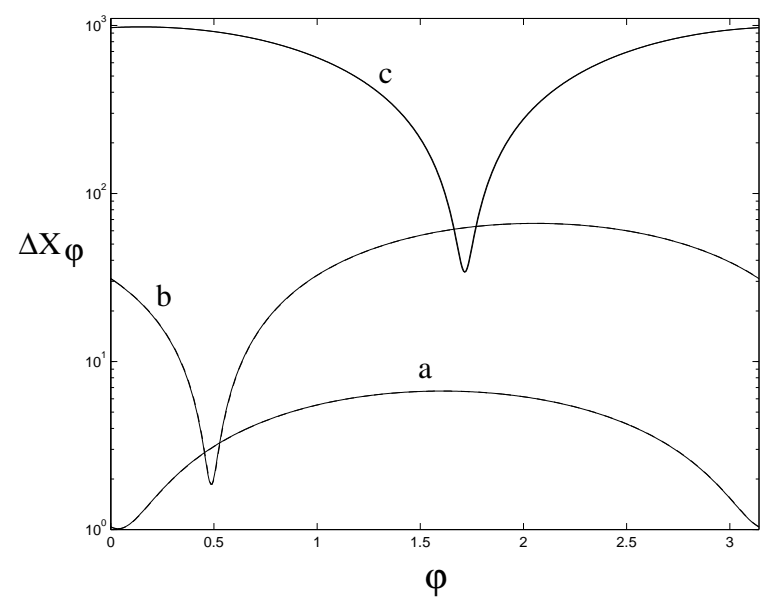

FIG. 4. The quadrature uncertainty $\Delta X_{\varphi}$ versus $\varphi$ for $k=3.3, \tau=0.01$, and various $|\alpha|:$ (a) $|\alpha|=10^{2}$, (b) $|\alpha|=10^{3}$, (c) $|\alpha|=10^{4}$. The difference between exact and approximate results (both of them shown in the plot) is almost invisible.

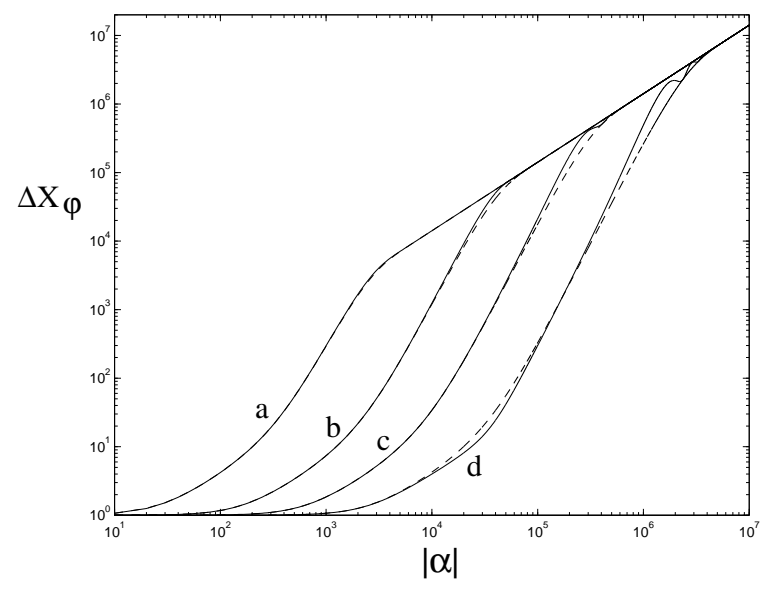

FIG. 5. The quadrature uncertainty $\Delta X_{\varphi}$ versus $|\alpha|$ for $k=3.3, \vartheta=0$, and various $\tau$ : (a) $\tau=0.05$, (b) $\tau=0.02$, (c) $\tau=0.01$, (d) $\tau=0.005$. Exact results are plotted with solid lines and approximate ones with dashed lines. 